\section{Critical limb ischaemia associated with chemotherapy for breast cancer}

\author{
W V Garrett BSc FRCS J R I Brown MRCS \\ D Pickering MRCP FRCR SM Andrews MS FRCS
}

J R Soc Med 2004;97:444-445

SECTION OF SURGERY, 6 JUNE 2003

The chemotherapeutic agents used for cancer have a wide range of reported side-effects but these have so far not included critical limb ischaemia. We have seen three cases in women being treated for ductal carcinoma of the breast.

\section{CASE HISTORIES}

\section{Case 1}

A woman of 56 was seen after her left foot had been cold and painful for 24 hours. In addition she said that for two months her fingers had been intermittently cold and blue. Her only risk factor for peripheral vascular disease was cigarette smoking, about ten a day. Recently an invasive ductal cancer of the breast had been treated with wide local excision and axillary clearance. One out of fifteen lymph nodes contained tumour but she was not thought to have metastatic disease elsewhere. She had undergone five cycles of cyclophosphamide, methotrexate and 5-fluorouracil (5FU) chemotherapy (CMF) and was taking tamoxifen. The 5-FU was administered as an intravenous bolus of $987.5 \mathrm{mg}$ at alternating one-week and three-week intervals. The last infusion had been 3 days earlier. On direct questioning, it was clear that the patient had first developed transient pain in her left foot after her first cycle of chemotherapy. When the pain recurred after the second cycle, deep venous thrombosis had been excluded by duplex scanning.

On examination her left foot was cold and pulseless and the third and fourth toes were dusky blue. A doppler signal was just audible over the posterior tibial artery but anklebrachial pressure indices were not recordable. An arteriogram revealed a normal aorta and iliac vessels but the left superficial femoral artery was attenuated with occlusion of the popliteal just above the joint line (Figure 1).

Department of Vascular Surgery, Maidstone District General Hospital, Hermitage Lane, Maidstone, Kent ME16 9QQ, UK

Correspondence to: Mr S M Andrews

E-mail: sam.andrews@lineone.net

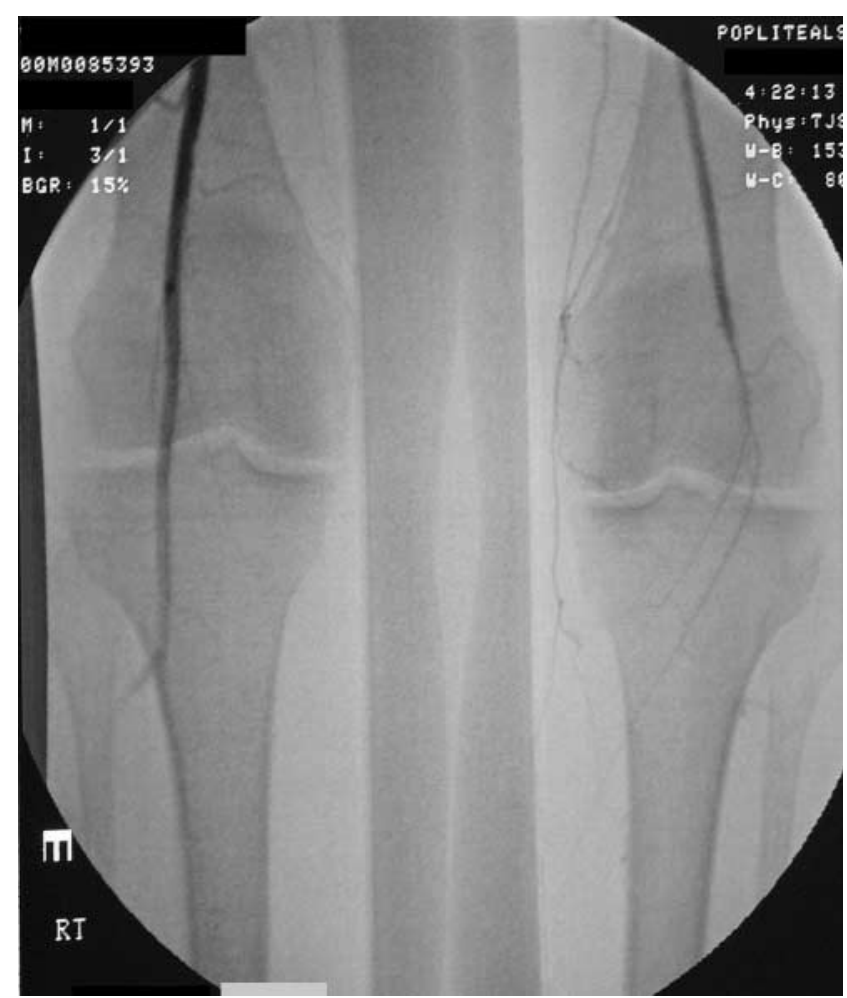

Figure 1 Arteriogram in case 1

The chemotherapy was stopped and she was treated with an intravenous bolus of 5000 units of unfractionated heparin followed by an intravenous heparin infusion of 24000 units over 24 hours. Three successive intravenous infusions of Iloprost (a prostacyclin analogue) $50 \mu \mathrm{g}$, in $250 \mathrm{~mL} 5 \%$ glucose, were given without improvement. Two days later a left chemical sympathectomy was performed and the foot became warmer though still painful. Aspirin $150 \mathrm{mg}$ daily was then started. Over the coming weeks she improved clinically and the fourth toe returned to normal. However, the pain remained troublesome. Further duplex scanning revealed no distal popliteal or trifurcation vessels but the posterior tibial vessel was seen to re-form just above the medial malleolus as a reasonable sized vessel with very damped flow. She underwent a reverse autologous long saphenous vein left popliteo-posterior tibial bypass and the third toe was amputated. Postoperatively she was heparinized and subsequently warfarinized. The graft remains patent at one year.

\section{Case 2}

A woman aged 46 was seen when three months of increasing pain in the fingers and toes, with occasional discoloration, culminated in a long episode of pain and redness in the right foot. She was undergoing cyclical intravenous CMF chemotherapy for invasive ductal 
carcinoma of the breast, which had been treated in the same way as in patient 1 , though in her case no lymph node involvement had been found and there were no risk factors for vascular disease. She too was taking tamoxifen. Her last 5-FU infusion had been 2 days earlier.

On examination she had normal femoral and popliteal pulses but distally no pulses could be felt and there were no audible doppler signals. She was admitted and treated with an intravenous bolus of 5000 units of unfractionated heparin followed by an intravenous heparin infusion of 24000 units over 24 hours. Transfemoral arteriography showed normal iliac, femoral, popliteal and trifurcation vessels but attenuation of the crural vessels at mid calf. She received five infusions of Iloprost $50 \mu \mathrm{g}$ in $250 \mathrm{~mL} 5 \%$ glucose. After a week there was complete clinical resolution. The chemotherapy was stopped and symptoms did not return. She remains well at eighteen months.

\section{Case 3}

A woman of 51 came to casualty with a cold, numb and painful right foot. She had no history of peripheral vascular disease and there were no apparent risk factors. As in cases 1 and 2 she was undergoing cyclical intravenous CMF chemotherapy after wide local excision of an invasive ductal carcinoma with axillary clearance (no lymph node involvement found). The 5-FU regimen was identical (last infusion 2 days earlier) and she too was taking tamoxifen. She was not thought to have metastatic disease. Clinically, pedal pulses were absent and the ankle-brachial pressure index at the ankle was 0.3 . She was admitted and treated with an intravenous bolus of unfractionated heparin 5000 units followed by an infusion of 24000 units over 24 hours. The following morning, the foot had greatly improved and the pressure index was 0.95. Since stopping chemotherapy the patient has had no further episodes.

\section{COMMENT}

A well recognized adverse effect of 5-FU is acute myocardial ischaemia or infarction. ${ }^{1,2}$ Coronary vasospasm has been postulated as the cause, ${ }^{3}$ with some in-vitro evidence suggesting the role of protein kinase C. ${ }^{4}$ This hypothesis remains unsubstantiated. ${ }^{5}$ In laboratory animals a direct cytotoxic effect of 5 -FU on vascular endothelium seems preventable by the use of antioxidant drugs. ${ }^{6}$ Although chemotherapy-associated Raynaud's phenomenon has been described, ${ }^{7}$ we have found no reports of critical limb ischaemia secondary to chemotherapy.

The three patients presented here had obvious limb ischaemia after chemotherapy. Might this have been a manifestation of the known tendency to thrombosis in malignant disease? We think not, because none had apparent residual disease at the time of the adjuvant chemotherapy. Moreover, cause and effect is suggested by the cyclical pattern of symptoms developing after drug administration and the improvement of symptoms after cessation of treatment.

In case 1 we postulate that the residual effects, requiring operative treatment, were due to thrombosis in small distal vessels. When symptoms of this kind arise, we recommend immediate cessation of chemotherapy.

\section{REFERENCES}

1 Keefe DL, Roistacher N, Pierri MK. Clinical cardiotoxicity of 5fluorouracil. J Clin Pharmacol 1993;33:1060-70

2 Lestuzzi C, Viel E, Picano E, Meneguzzo N. Coronary vasospasm as a cause of effort-related myocardial ischemia during low-dose chronic continuous infusion of 5-fluorouracil. Am J Med 2001;111:316-18

3 Kleiman NS, Lehane DE, Geyer CE Jr, Pratt CM, Young JB. Prinzmetal's angina during 5-fluorouracil chemotherapy. Am $\mathrm{J}$ Med 1987;82:566-8

4 Mosseri M, Fingert HJ, Varticovski L, Chokshi S, Isner JM. In vitro evidence that myocardial ischemia resulting from 5-fluorouracil chemotherapy is due to protein kinase $\mathrm{C}$-mediated vasoconstriction of vascular smooth muscle. Cancer Res 1993;53:3028-33

5 Becker K, Erckenbrecht JF, Haussinger D, Frieling T. Cardiotoxicity of the antiproliferative compound fluorouracil. Drugs 1999;57:475-84

6 Kinhult S, Albertsson M, Eskilsson J, Cwikiel M. Effects of probucol on endothelial damage by 5-fluorouracil. Acta Oncologica 2003;42:304-8

7 Papamichael D, Amft N, Slevin ML, D'Cruz D. 5-Fluorouracil-induced Raynaud's phenomenon. Eur J Cancer 1998;34:1983 